\title{
Geschichten über Metall, Holz und Schnee
}

Zur narrativen Bedeutung von digitalen Materialien

Histoires sur le métal, le bois et la neige : la dimension narrative des matériaux digitaux

Stories about Metal, Wood and Snow - Towards the Meaning of Digital Materials

\section{Markus ENGELNS}

\section{(2) OpenEdition}

\section{Journals}

Édition électronique

URL : http://journals.openedition.org/ceg/3590

DOI : 10.4000/ceg.3590

ISSN : 2605-8359

Éditeur

Presses Universitaires de Provence

\section{Édition imprimée}

Date de publication : 15 novembre 2018

Pagination : 95-106

ISBN : 979-10-320-0183-7

ISSN : 0751-4239

Référence électronique

Markus ENGELNS, « Geschichten über Metall, Holz und Schnee », Cahiers d'Études Germaniques

[Online], 75 | 2018, Online erschienen am: 25 April 2020, abgerufen am 28 November 2020. URL

http://journals.openedition.org/ceg/3590 ; DOI : https://doi.org/10.4000/ceg.3590 


\title{
Geschichten über Metall, Holz und Schnee \\ Zur narrativen Bedeutung von digitalen Materialien
}

\author{
Markus ENGELNS \\ Universität Duisburg-Essen
}

\section{Digitale Materialität}

Viele Videospiele geben sich auf der Darstellungsebene äußerste Mühe, Materialien wie Holz, Stein, Metall, Schnee oder Wasser, aber auch Stoffe, Haare, Haut und sogar Knochen möglichst realistisch abzubilden. Dabei sollten die jeweiligen simulierten Materialien bestenfalls nicht nur das Aussehen, sondern auch die physikalischen Eigenschaften ihrer analogen Pendants aufweisen; Holz könnte brennen, Stoff im Wind wehen und Steine sollten im Wasser untergehen. Videospiele simulieren diverse Materialien nicht nur, um einen möglichst realistischen oder alltagsbezogenen Eindruck zu erwecken, sondern auch, um Spielprozesse zu steuern und zuweilen sogar ganze Geschichten über sie zu erzählen. Der folgende Beitrag will dem begegnen, indem er das Abenteuerspiel Syberia ${ }^{1}$, das Materialien nicht nur darstellt, sondern nachhaltig in eine komplexe Narration einbaut, untersucht. Zu fragen ist, 1.) wie die digitalen Spiele den Eindruck von Materialität überhaupt erst herstellen, 2.) welche Funktionen diese Darstellung übernimmt und 3.) inwiefern das Material Teil einer Erzählung werden kann. Die These lautet dabei, dass die Darstellung von Materialien in Videospielen insbesondere aus der Sicht der Technik betrachtet werden muss: Zentral ist die Textur, eine Materialtapete, die auf digitale Objekte übertragen werden kann. Solche Texturen bilden keine einfachen mimetischen Repräsentationen, sondern Simulationen, die einige Verhaltensweisen ihrer Vorbilder aus der analogen Alltagswelt nachbilden. Ein Rekurs auf Gernot Böhmes Überlegungen zur Spanplatte ${ }^{2}$ kann diese Überlegungen noch weiter ausdifferenzieren. Indem Böhme die Spanplatte in einem Spannungsverhältnis zwischen dem äußerlich beklebten Holzmöbel als Ware und als Designobjekt auf der einen und minderwertig zusammengeklebten Holzspänen im Inneren auf der anderen Seite situiert, verschiebt er den Fokus der Betrachtung von der Materialität hin zur äußerlichen Anmutung des Materials, das im Inneren keine

1. Microïds, Benoît Sokal, Syberia, Atari, 2001. Fortgesetzt 2003 und 2017.

2. Gernot Böhme, Atmosphäre. Essays zur neuen Ästhetik, Frankfurt a.M., Suhrkamp, 2013, S. 57. 
Entsprechung mehr hat. Syberia bezieht zu solchen Überlegungen Stellung, indem es die europäische Geschichte in einem Übertrag von der materiellen Arbeit des 20. Jahrhunderts hin zu einer digitalen Arbeit des 21. Jahrhunderts fokussiert, auch, um deren Zusammenfall zu beschreiben. Dabei gibt sich das Spiel alle Mühe, durch äußere Erscheinungen der Material-Textur in Form von Naturmaterialien, aber auch Rost und Erosion die vergehende Zeit dieser Historie sichtbar zu machen. Um diese Thesen besser plausibilisieren zu können ist es sinnvoll, den Blick auf die Textur als technisches und narratives Element zu richten.

\section{Funktionen von Materialien in Videospielen}

Die sehr unterschiedlichen Funktionen von Materialien können besonders leicht anhand eines Beispiels aus Syberia erläutert werden. Das Spiel, das maßgeblich von dem belgischen Comicschaffenden Benoît Sokal konzipiert wurde, handelt von der jungen New Yorker Anwältin Kate Walker, die für ihren ersten großen Auftrag in das fiktive französische Alpendorf Valadilène reist, um dort den Verkauf einer Manufaktur, die seit dem 19. Jahrhundert roboterhafte Automaten mit menschlichen Charakterzügen herstellt, an einen internationalen Spielzeughersteller abzuwickeln. Doch als Kate Walker in Frankreich ankommt, erfährt sie, dass die Besitzerin verstorben ist. Komplizierter wird der Fall, als im letzten Schreiben der Verstorbenen die Rede von einem lang verschollenen Bruder und Erben ist, der nun aufgefunden werden soll. Hans Voralberg, der Erbe und seines Zeichens genialer, aber nach einem Unfall in der Jugend eher kindlicher Konstrukteur teils raffınierter Konstruktionen, reist seit Jahrzehnten aufgrund eines Bruchs mit seiner Familie durch Europa. Er ist dabei auf den Spuren einer Legende, den Yukol, einem Stamm in Sibirien, der vorgeblich die letzten noch lebenden Mammuts auf der bisher unentdeckten Insel Syberia kultiviert. Kate Walker bleibt demnach nichts anderes übrig, als sich mit einem von Hans Voralberg konstruierten Zug auf eine Reise zu begeben, die eigentlich die verstorbene Schwester hätte antreten sollen. Da Hans den Zug so konstruiert hat, dass er in jedem Bahnhof, in dem auch Hans auf seiner Reise Halt gemacht hat, stehenbleibt, ist es das erste Ziel des Spiels, in den verschiedenen Stationen - Valadilène in Frankreich, Barockstadt in Deutschland, Komkolzgrad, Aralbad und Romansburg in Russland und von da an durch die sibirische Eiswüste den Zug wieder zum Fahren zu bringen. Hierzu muss Kate Walker mit den teils skurrilen Bewohnern der jeweiligen Ortschaften sprechen, Aufgaben für sie erledigen und in den allermeisten Fällen mechanische Rätsel lösen, die dazu führen, dass der Zug seinen Weg fortsetzen kann. Dabei weist das Spiel insgesamt vier komplexe Erzählebenen auf: Die erste Ebene handelt von Kate Walkers Reise in den Osten, auf der sie mehrfach in verschiedenen europäischen Städten haltmacht, weil der Zug jeweils nicht weiterfahren kann. Hier erlebt Kate Walker in kleinen Handlungsepisoden Stationen aus Hans Voralbergs Leben, sie lernt Figuren kennen, die ihn getroffen haben und sie bedient seine raffinierten Erfindungen, damit sie die Reise fortsetzen kann. Stilistisch verwendet das Spiel 
hier Sehnsuchtsmotive der Romantik, indem es Kate Walker auf die Reise einer anderen schickt. Insofern verwundet es nicht, dass sie in den Szenen, in denen der Zug fährt, die Natur, den Sonnenuntergang und die Strecke samt Tunneln und Gleisen sehnsüchtig aus dem Fenster betrachtet. Die Handlungsorte vermengen dabei einen französischen Jugendstil mit filigranen Metallarbeiten, die teils organisch und floral aussehen und durch Rekurse auf die industrielle Revolution mit Fabrikhallen, Fließbändern und Werkzeugen kontrastiert werden. Die zweite Ebene erzählt Kate Walkers zunehmende Entfremdung von ihrem Verlobten, ihrer Arbeit und ihrer besten Freundin in New York, die in diversen Telefonaten immer wieder eingeschaltet werden. Die dritte Erzählebene beschäftigt sich mit der Biografie von Hans Voralberg, dem Bruch mit seiner Familie und der Geschichte der Manufaktur Voralberg im 20. Jahrhundert. Schließlich berichtet die vierte Erzählebene die Geschichte Europas im 20. Jahrhundert, genauer von der industriellen Revolution, vom Zweiten Weltkrieg, dem geteilten Deutschland sowie dem Aufstieg und Fall der Sowjetunion.

In der ausgewählten Beispielszene vom Anfang des Spiels soll Kate Walker - und mit ihr auch der Spieler - in der Manufaktur Voralberg für den bisher nicht fertigstellten Zugführer-Automaten Oskar neue Beine konstruieren, damit dieser seinen Dienst antreten kann. Der anthropomorphisierte Oskar händigt Kate Walker dazu eine Schlüsselkarte aus. Der Spieler muss daraufhin zunächst die stillgelegte Fabrik wieder in Betrieb nehmen, um anschließend im Materiallager das korrekte Holz auszuwählen und den Fertigungsprozess in Gang zu setzen. Das Problem lautet in dieser Situation, mit Hilfe einiger in der Spielwelt verstreuter Informationen die richtige Holzart zu finden. Diese eigentlich recht unspektakulär klingende Aufgabe verdeutlicht einige der in diesem Zusammenhang relevanten Funktionen von Materialien in Videospielen, da sie simulativ-spielprozessuale, aber auch narrative Funktionen erfüllt.

Zunächst einmal befindet sich Kate Walker in einem Materiallager, in dem verschiedene Holzsorten auf ihre Verwendung warten. Hat der Spieler zuvor einen Prospekt in der Werkstatt gefunden, in dem die Färbung des für den Automaten verwendeten Holzes beschrieben wird, kann er relativ leicht die korrekte Einstellung vornehmen. Ohne diese Informationen kann es aber auch passieren, dass der Spieler die falsche Sorte wählt, woraufhin Oskar sie bittet, es erneut zu probieren. Das Materiallager ist dabei das perfekte Bild für Prozesse der Simulation in Videospielen: Diese basieren nämlich zunächst nicht auf einem mehr oder weniger zeitlich bzw. syntagmatisch geordneten Zeichensystem. Vielmehr stellen sie Datenbanken dar, in denen verschiedene Dateneinträge - Grafiken, Filmsequenzen, Sounds, Musik, Texteinträge oder eben verschiedene Holzsorten - vorrätig gehalten werden, die bei Bedarf abgerufen werden können. Sie bilden also „Ausgangsobjekte, die sequentiell oder parallel wiederholbar sind“3. Das Spiel kann demnach zweierlei: Einerseits bietet es

3. Leif Rumbke, „,P1 Ready؛ Das klassische Shoot'em Up als kinetische Konfiguration“, in Mattias Bopp, Serjoscha Wiemer, Rolf F. Nohr (Hrsg.), Shooter. Eine multidisziplinäre Einführung, Münster, LIT, 2009, S. 43-74, hier S. 49. 
verschiedene Holzsorten aus der Datenbank heraus an, andererseits realisiert es eine Holzart als eine, deren Wahl das Spiel voranschreiten lässt, wohingegen die übrigen in Sackgassen führen und einen Neustart der Aufgabe erfordern. Solche Datenbank-, Kausal- und Verweissysteme hat maßgeblich Espen Aarseth unter dem Begriff des „ergodic text“" näher erläutert. Ergodische Texte sind vor allem dadurch gekennzeichnet, dass sie aus mehreren Texteinträgen bestehen, die Verlinkungen untereinander aufweisen. Die Aufgabe des Rezipienten innerhalb solcher Textdatenbanken ist es, die eigentliche Abfolge und Struktur des Textes erst dadurch herzustellen, dass sie bestimmten Verlinkungen folgen und andere dabei auslassen, um so einen eigenen Weg durch das Textgebilde zu finden. Deshalb ist der Begriff, ergodic ‘ auch ein Lehnwort aus, ergon ' (Arbeit) und, hodos (Weg), um den Aufwand des Rezipienten zu fokussieren, den Text überhaupt erst herzustellen. Die allermeisten Videospielmodelle fußen in mehr oder weniger konkreter Weise auf Aarseths Überlegungen und subsumieren die Datenbank sowie die dazugehörigen Verlinkungen mitsamt den damit einhergehenden Spielmöglichkeiten unter dem Begriff der Simulation, die auch zusätzlich für die Gestaltung von Weltregeln und die korrekte Darstellung von physikalischen Effekten zuständig ist. ${ }^{5}$ Die Simulation steuert somit nicht nur die Verwendbarkeit des Materials durch den Spieler, sondern auch dessen Eigenschaften wie die Brennbarkeit, den Bezug zur Schwerkraft oder das Verhalten im Wasser.

Die Elemente der Simulation sind aber weitestgehend abstrakt, handelt es sich hierbei doch um ein informatisches System, das Eingaben verarbeiten, sich an den Spieler anpassen kann und Entscheidungsmöglichkeiten bereithält. Deswegen ist von Seiten der frühen game studies vertreten worden, dass klassische Medien auf dem Prinzip der Repräsentation - gedacht als ein Prozess, bei dem Mitglieder eines Sprachsystems auf dieses zugreifen, um „Bedeutung zu produzieren“6 basieren, wohingegen Videospiele Simulationen seien. ${ }^{7}$ Simulation meint in diesem Zusammenhang die Modellierung eines abgeschlossenen mathematischen Kausalsystems, in das Werte eingefüttert werden können und diese nach den je einprogrammierten Regeln verrechnet werden. Beispielhaft sei etwa die Wettersimulation genannt, die verschiedene Daten aus der Alltagswelt verrechnen

4. Espen J. Aarseth, Cybertext. Perspectives on Ergodic Literature, Baltimore u.a., The Johns Hopkins University Press, 1997, S. 1; vgl. hierzu auch Marie-Laure Ryan, Narrative as Virtual Reality. Immersion and Interactivity in Literature and Electronic Media, Baltimore/ London, The Johns Hopkins University Press, 2001.

5. Vgl. hierzu u.a. Hans-Joachim Backe, Strukturen und Funktionen des Erzählens im Computerspiel. Eine typologische Einführung, Würzburg, Königshausen \& Neumann, 2008, aber auch, Markus Engelns, Spielen und Erzählen. Computerspiele und die Ebenen ihrer Realisierung, Heidelberg, Synchron, 2014.

6. Christian Riedel, „Waffen, Konventionen, Mythen. Versuch einer kulturellen Deutung verschiedener Lesarten des Waffenzeichens im Shooter-Spiel“, in Bopp, Wiemer, Nohr (Hrsg.), Shooter. Eine multidisziplinäre Einführung, S. 247-266, hier S. 249 und Stuart Hall, „The Work of Representation“, in ders., Representation. Cultural Representations and Signifiying Practices, London, Sage, 1997, S. 13-74.

7. Gonzalo Frasca, Simulation 101. Simulation versus Representation, 2001, [http://www.ludology. org/articles/sim1/simulation101.html], Stand: 1. September 2017. 
kann, um anschließend die Wahrscheinlichkeit der Entwicklung des Wetters in den kommenden Tagen wiederzugeben. Obwohl der Fokus auf die Simulation als immanenter Teil von Videospielen einige ihrer spezifischen Merkmale zu erklären vermag, etwa die Interaktivität, kann der Begriff der Repräsentation nicht vollends aufgegeben werden. Auf der Ebene der Simulation ist das in der Beispielaufgabe verwendete Holz zunächst nicht mehr als eine abstrakte Zahl, die keine sozial oder kulturell identifızierbare Dimension hat. Deshalb wäre es mit einem solchen System streng genommen auch möglich, Aufgaben in völlig anderen Kontexten ablaufen zu lassen. Die Wahl des Holzes in Syberia könnte also auch eine Wahl von Nahrungsmitteln oder in Actionspielen die Wahl von Waffen sein. Bedeutung in einem kulturellen Sinne erhält die Zahl nur, indem sie auf einer anderen Ebene als Holz sichtbar gemacht wird. Hierzu ist der Begriff der Repräsentation notwendig.

Hans Joachim Backe führt in seiner Studie zu Computerspielen aus, dass der semantische Gehalt in Videospielbildern aus der Spielperspektive in der Regel weniger wichtig ist als spielprozessuale und simulative Überlegungen. Zu einem Spiel, das in einem Abschnitt in einer Gelddruckerei stattfindet, argumentiert er, dass die kapitalistischen und wirtschaftlichen Implikationen weniger bedeutsam seien als technische Probleme der Darstellbarkeit:

Im Computerspiel treten metaphorische Überlegungen hinter die Spielbarkeit, Effizienz und Relevanz der Umgebung zurück. [...] Papierstapel oder -rollen sind außerdem einfache geometrische Formen [...], was zur Effizienz der Spielumgebung beiträgt, sie also relativ wenig Computerressourcen verwenden läßt. ${ }^{8}$

Diese demnach eher niedrige Priorität der Darstellungsebene des Spiels heißt aber im Umkehrschluss nicht, dass Papierstapel oder in diesem Fall die Wahl des Holzes nicht doch auf dem Prinzip der Repräsentation basieren. Die Identifikation des simulativ noch abstrakten Gegenstandes als Holz verleiht dem jeweiligen Objekt einen spezifischen semantischen Gehalt in der Rezeption. Deshalb ist es sinnvoll, neben der simulativen Basisebene eine zweite einzuziehen, die die eher informatischen Realisierungen der Simulation mit soziokulturell identifizierbaren Repräsentationen versieht. Das bedeutet, dass das Spiel seine Gegenstände soziokulturell erfahrbar macht, indem es Grafiken, Sounds, etc. verwendet, um Materialien, menschenähnliche Figuren, Häuser und dergleichen mehr als solche überhaupt erst zu realisieren. Syberia gibt sich bei diesem Prozess besondere Mühe: In der Produktionssequenz, die minutiös zeigt, wie ein mechanischer Arm das ausgewählte Holz erst aus dem Regal zieht, es dann auf ein mechanisches Förderband legt, wo es von Automaten gedrechselt und durchbohrt wird, um abschließend als zwei neue Füße vom Band zu fallen, sind die Qualitäten des Materials als Repräsentationen deutlich sichtbar. Das Holz ist teilweise gesprungen und gemasert, das Metall ist verrostet, Geräusche werden verwendet, um die Reibung des Metalls, die Bearbeitung des Holzes anzuzeigen.

8. Backe, Strukturen und Funktionen, S. 99, FN 178. Vgl. hierzu auch Markku Eskelinen, The Gaming Situation, 2001, [http://www.gamestudies.org/0101/eskelinen/], Stand: 1. September 2017. 
Die Repräsentation des Materials ist in dieser Passage augenscheinlich so wichtig, dass diverse technische Maßnahmen ergriffen werden, um sie verständlich zu machen. Durch Repräsentation entsteht aber nicht nur der denotative Eindruck von Materialität; vielmehr zieht das Spiel dadurch auch zahlreiche Konnotationen ein, die im Rahmen einer narratologischen Betrachtung von Bedeutung sein können. Bevor diese aber genauer verfolgt werden kann, ist es sinnvoll, zunächst einen Blick auf die Textur als Teil des Spiels zu werfen.

\section{Die Textur als eine besondere Spanplatte}

Die Textur wurde und wird als Teilelement von Videospielen bereits seit den 1990er Jahren vor allem in dreidimensionalen Spielen verwendet, um den Anschein von Materialität herzustellen. Dreidimensionale Objekte, Figuren und Räume werden dabei zunächst als abstrakte und animierbare Drahtgittermodelle modelliert, die anschließend einen Materialanstrich erhalten, der als Textur bezeichnet wird. Beispielhaft sei Kate Walker als eine auf diesem Weg modellierte Figur genannt, die nach der Herstellung von Körperkonturen und Animationen mehrere Texturen erhält, die ihre Haut und ihre Kleidung darstellen. In Syberia ist die Textur maßgeblich dafür zuständig, den meist optischen, manchmal auch auditiven Eindruck von Materialität herzustellen: Steinböden erzeugen beim Laufen andere Töne als Holzdielen und älteres Metall kann von neuerem durch Rost unterschieden werden. In neueren Spielen allerdings übernimmt die Textur noch deutlich mehr Funktionen, indem sie beispielsweise Lichtreflexionen, Schattenwürfe sowie Reaktionen von Gegenständen auf Regen oder andere Witterungen generiert. Die Textur hat somit simulative Funktionen, weil sie physikalische Regeln im Spiel mitrealisiert. Sie hat zudem auch soziokulturelle Funktionen, indem sie die abstrakten Digitalobjekte identifizierbar macht.

Durch die Textur entsteht ein Spannungsverhältnis zwischen dem hohlen Drahtgittermodell im Inneren des Objekts und dem reich ausstaffierten, weil texturierten Äußeren. Dieses Wechselverhältnis kann mit Hilfe von Gernot Böhmes Überlegungen zur Materialästhetik noch einmal genauer gefasst werden. Böhme geht davon aus,

daß eine Atmosphäre, die ja als solche ein diffuses Ganzes, wenngleich von erkennbarer und wiedererkennbarer Charakteristik ist, durch gegenständliche und auch objektiv identifizierbare Elemente erzeugt werden kann, und zwar in der Regel sogar sehr wenige. ${ }^{9}$

$\mathrm{Zu}$ solchen Elementen gehören vor allem Gegenstände im Raum, die handwerklich aus besonderen Materialien hergestellt werden und durch ihre Form eine Funktion erhalten. ${ }^{10}$ Form und Material eines Gegenstands stehen dabei in einem unterstützenden oder in einem einander widersprechenden

9. Böhme, Atmosphäre, S. 78.

10. Ibid., S. 50. 
Spannungsverhältnis, das er als Materialästhetik fasst. Die Materialästhetik ist dabei kein semiotischer Text. Vielmehr spiegelt sie den „gesellschaftliche[n] Charakter" wider ${ }^{11}$, der Einfluss des Objekts auf die Atmosphäre auch in Bezug auf seine gesellschaftliche Verortung beschreibt. ${ }^{12}$ Die Atmosphäre ist bei Böhme also auch ein Oberflächenphänomen, weil sich Baumaterialen im Inneren deutlich von ihrem Äußeren unterscheiden können. Die Spanplatte, so führt er aus, sehe durch Oberflächentexturen wertvoll aus. Im Inneren besteht sie aber aus einem gänzlich anderen Material. ${ }^{13}$ Diese Differenz ist dabei sowohl ästhetischen Überlegungen, die das Äußere bestimmen, als auch ökonomischen Überlegungen entsprungen, die das eher minderwertige Innere der Spanplatte begründen. „Es gibt eine ästhetische Ökonomie, und zwar nicht nur im Sinne einer Bestimmung der Ästhetik durch die Ökonomie, sondern auch umgekehrt der Ökonomie durch die Ästhetik“ ${ }^{14}$, weil das Äußere der Spanplatte maßgeblich an der Raumatmosphäre mitwirkt und das ökonomisierte Innere umschließt. Gesellschaftliche Arbeit bzw. Handwerk sei demnach oft „ästhetische Arbeit oder Inszenierungsarbeit“" ${ }^{15}$. Die Textur ist diesen Überlegungen folgend eine besondere Form der Spanplatte, bei der das Innere des Gegenstands nicht einfach nur minderwertiger, sondern schlicht nicht mehr vorhanden ist - es bildet „nur“ den Hohlraum zwischen den Drahtgittern des 3D-Modells. Dadurch wird das von Böhme skizzierte Spannungsverhältnis größer, weil die ressourcenschonende und mithin ökonomische Leere im Inneren im Spannungsverhältnis zur Darstellung von Materialität durch die Textur auf dem Äußeren des Gegenstandes steht. Das bedeutet, dass die Darstellung von Materialien in Videospielen immer auch aus einer ökonomischen Sicht betrachtet werden muss, wie die folgenden Überlegungen zu Syberia zeigen.

\section{Spielbare Material-Geschichten}

Von besonderer Bedeutung für die Analyse von Materialien in Syberia ist insbesondere die vierte Erzählebene, in der es um die Geschichte Europas geht. Dabei fallen der historische und der räumliche Fortschritt chronotopisch im Spiel zusammen: Je weiter Kate Walker nach Osten reist, desto weiter schreitet sie im 20. Jahrhundert voran. ${ }^{16}$ Dementsprechend wird Valadilène beherrscht von Jugendstil-Fassaden, die mit Motiven der industriellen Revolution - Manufakturen, Automaten, Fließbänder - kombiniert wurden. Eine im Spiel befındliche fiktive Broschüre berichtet von den ersten Marionetten, die bereits im 13. Jahrhundert im Ort hergestellt wurden, bis zum „goldenen Zeitalter

11. Ibid., S. 53.

12. Ibid., S. 52.

13. Ibid., S. 57.

14. Ibid, S. 63.

15. Ibid.

16. Vgl. Michail M. Bachtin, Chronotopos, Frankfurt a.M., Suhrkamp, 2008. 
der Stadt“ am Ende des 19. und Anfang des 20. Jahrhunderts. ${ }^{17}$ Im weiteren Verlauf des Spiels wird darüber hinaus deutlich, dass es im Wald hinter der Stadt eine Höhle gibt, in der steinzeitliche Wandbilder des Menschen zu finden sind. Valadilène entspricht somit der Geschichte Europas von der Steinzeit bis ins 20. Jahrhundert hinein. In der Spielgegenwart am Anfang des 21. Jahrhunderts wird Valadilène dementsprechend auch als ein verlassener Ort dargestellt, dessen Produktionsstätten und Kultur am Anfang der Moderne stehengeblieben sind. Den Zusammenfall von Örtlichkeit und Geschichte erzählt Syberia dabei einerseits über einige im Spiel befindliche erläuternde Texte wie Tagebücher, andererseits aber vor allem über die bildliche Darstellung der jeweiligen Städte und ihrer Konstruktionen. Dementsprechend weist der Ort als Brücke zwischen Vormoderne und Moderne zunächst Gebäude und Objekte aus Metall und Stein auf, die zur industriellen Revolution zu zählen sind. Im Hintergrund allerdings fließen Bäche durch dichte Wälder, die später auch im Rahmen der Suche nach der steinzeitlichen Höhle betreten werden. Vom Material her gedacht veranschaulicht das Spiel somit den Ausgang des Menschen aus seinen natürlichen Bezügen und den Übergang zum Kulturwesen anhand der Verwendung von Holz, Stein und Metall. Tatsächlich ist Valadilène der einzige Ort, der im Rahmen dieser Geschichte Europas besucht wird, der überhaupt ein Stück weit über eine nichtkultivierte Natur verfügt.

Diese spezifische Erzählform, die meist ohne Worte auskommt und nur durch das Zusammenspiel der von Bildelementen, Räumen und der Rezeption zustande kommt, hat Henry Jenkins treffend als „spatial storytelling“ ${ }^{18}$ bezeichnet. Syberia nutzt sie intensiv, um kulturelle und politische Gegebenheiten sichtbar zu machen, ohne sie textuell benennen zu müssen. Höhepunkt dieser Entwicklung ist Kate Walkers Aufenthalt in Barockstadt, der den Zweiten Weltkrieg, die DeutschDeutsche Teilung und den 0st-West-Konflikt nahezu gänzlich ohne Worte evoziert: Barockstadt ist eine alte, inzwischen wie alle Orte im Spiel verlassene Universitätsstadt, deren Ursprünge dem sprechenden Namen der Stadt nach im 16. Jahrhundert verortet werden kann. Somit steht der Ort auch für ein Land der ,Dichter und Denker. Barockstadt ist von einer schweren Mauer gen Osten abgeschottet, hinter der eine - den Legenden im Ort nach - unwirtlich trostlose Steppe liegt, in der ein feindliches Reiterheer stationiert ist, das nur auf den Befehl zum Angriff wartet. Ähnlichkeiten zur Berliner Mauer sind also gegeben, wobei diesmal der Antifaschistische Schutzwall vom Westen konstruiert wurde, aus Angst, der Osten könnte einfallen. Die Mauer und die Universität werden dabei von einem recht langen Weg verbunden, der von zerbombten Häuserruinen gesäumt ist, die vom Spiel unkommentiert bleiben, dabei aber fatal an die ausgebrannten Häuser einer Bombardierung durch Flugzeuge erinnern. Das Spiel evoziert hier die Geschichte Deutschlands vom Barock über die industrielle

17. Microïds, Benoît Sokal, Syberia.

18. Henry Jenkins, „Game Design as Narrative Architecture“, in Noah Wardrip-Fruin, Pat Harrigan (Hrsg.), First Person. New Media as Story, Performance, and Game, Cambridge (Mass.)/ London, The MIT-Press, 2004, S. 118-130, hier S. 122. 
Revolution über den Zweiten Weltkrieg (wobei die Shoa ausgespart wird) bis hin zur deutschen Teilung.

Auch hier wird die Geschichte Europas maßgeblich in Bildern repräsentiert und zumeist gar nicht oder kaum kommentiert. Die Texturen der jeweiligen Objekte bzw. die Materialien zeigen dabei nicht nur den Verlauf von Zeit durch Rost, Zerstörung und anachronistische Bauformen auf, sondern loten über die verwendeten Materialien auch eine kleine Kulturgeschichte des Westens aus, wobei alle Materialien spezifische semantische Bedeutungen erhalten: Holz und Stein können als natürliche Ressourcen verwendet werden, aus denen später Geräte und Häuser gebaut werden. Metall steht für die Umwandlung solcher Naturprodukte in kulturelle Gegenstände. Schnee, der ab dem Ende des ersten Teils der Reihe wichtig wird, dient dazu, den aufgewirbelten Sand der Vergangenheit des 20. Jahrhunderts wieder zu begraben: In dem Kurort Aralbad lernt Kate Walker eine alternde, vom Publikum längst vergessene Operndiva kennen, die sie zu einem allerletzten Konzert verhilft, nach dem ein „lange[r] Winter" ${ }^{19}$ beginne. Am Ende des ersten Teils fängt es schließlich an zu schneien: „Der Schnee vertreibt den Wind und Salz und Sand bleiben am Boden. Die Luft wird rein und beruhigend. Ein ideales Wetter für Spaziergänge“. ${ }^{20}$ Der Schnee überdeckt die Spuren der Vergangenheit und lässt das 20. Jahrhundert erschlaffen. Zudem ist dieses Erschlaffen auch im Motiv des Zuges, mit dem Kate Walker durch Europa reist, festgehalten. Diese mechanisch raffınierte Konstruktion basiert nämlich auf einem Federmechanismus, der in jedem Bahnhof der Reise neu aufgezogen werden muss. Insofern ist der Zug als Reisemittel, wie auch als Maschine ein Bild für das zusehends erschlaffende Jahrhundert der Mechanik, das durch Kate Walkers Reise ein letztes Mal wieder aufgezogen wird und dann anschließend vollends erstarrt. Insofern erzählt Syberia eine postindustrielle Verfallsgeschichte, deren Motiv die Rolle des Menschen in einer Welt der vollautomatisierten Arbeit ist, aus der aber zunächst nichts mehr erwächst, weil die Mechanismen abgeschaltet sind und die wenigen Übriggebliebenen ihren letzten Träumen nachhängen, ohne sie erfüllen zu können. Das Spiel zeigt somit im Grunde das Ende von Kulturation, Fortschritt und Moderne. Die Bilder dieser Geschichte sind die immer wieder fokussierten Hände der anthropomorphen Automaten, die mal Züge lenken, mal Manufakturen steuern oder Klaviere spielen, und der Zug selbst, dessen Sprungfeder immer wieder erschlafft und aufgezogen werden muss.

In diesem Zusammenhang ist das eingangs ausführlich vorgestellte Rätsel um die Beine des Zugführerautomaten Oskar von Bedeutung, zeigt das Spiel hier doch, wie es das Material und dessen Lager mit zahlreichen Diskursen auflädt. Das Materiallager, in dem das Holz zur Produktion der Füße aufbewahrt wird, mitsamt der Werkstatt und der dazugehörigen automatischen Fließbänder ist nämlich nicht einfach nur ein Spielort. Vielmehr verweist es aufgrund zahlreicher Merkmale auf diverse technologische Punkte: So fällt auf, dass das Materiallager einer verlassenen Produktionshalle entspricht, die als Verweis auf

19. Microïds, Benoît Sokal, Syberia.

20. Ibid. 
einen postford'schen Arbeitsprozess gelten kann. Wäre die Szenerie dadurch wieder in der industriellen Revolution anzusiedeln, durchbricht insbesondere die Lagerverwaltung diese zeitliche Verortung. Das Material holt Kate Walker nämlich nicht selbst aus dem Lager. Vielmehr kommt ein metallener Greifarm zum Einsatz. Diese Art der Lagerverwaltung erinnert eher an digital gesteuerte Technologien, die aus den Lagerhäusern von Online-Versandhandeln bekannt sind. Deshalb referiert das Spiel in einem kurzen Dialog zwischen Kate Walker und Oskar auf Digitaltechnologien: Als er sie anweist, das Fußmodell XZ2005_B zu produzieren, weil das A-Modell Fehler aufweise, erkundigt sich Kate Walker danach, ob er einen Bug habe - was im Fachjargon von Videospieldesignern einen technisch bedingten Fehler im Spiel bezeichnet. Oskar gibt zwar an, dass Automaten keine Bugs, sondern nur „eigenwillige Funktionsweisen“ hätten. ${ }^{21}$ Dennoch ist damit der Übertrag auf eine Reflexion von Videospielstrukturen bzw. ein Hinweis auf eine noch rudimentär ausgeprägte Metareflexion gegeben. Tatsächlich hat das Materiallager strukturelle Gemeinsamkeiten mit der Simulation als Datenbank, deren Einzeleinträge ausgelesen werden müssen - nicht umsonst erinnert der Greifarm des Materiallagers an den Arm, der die Festplatte des Computers verwaltet. Das Material ist hier somit nicht nur ein Gegenstand der Simulation, sondern auch ein Mittel zur Reflexion von Simulationen.

Der Verweis auf digitale Technologien des 21. Jahrhunderts ist in Syberia durch eine Dominanz des patriarchalen Produzenten im 20. Jahrhundert bestimmt: Die Manufaktur Voralberg und die Werkstatt verweisen in vielfacher Hinsicht auf männlichen Produktionsanspruch, auf eine göttliche Schaffenskraft. Alle Automaten sind männlich gezeichnet. Darüber hinaus wird der Gründer des Familienbetriebes, Charles Voralberg, in der Firmenbroschüre auf einem Portrait aus dem 17. Jahrhundert als Vater mit einer Marionette im Arme gezeigt, die einem Baby gleicht. Zudem entspricht die Darstellung der Werkstatt, in der Oskar unfertig an einer Strebe an der Decke hängt, der aus Kenneth Branaghs Frankenstein-Verfilmung stammenden Werkstatt, in der die berühmte Kreatur aus Leichenteilen zusammengebaut wird. ${ }^{22}$ Außerdem findet Kate Walker in der Kirche von Valadilène ein Kruzifix, das Jesus Christus als Automaten darstellt. Das 20. Jahrhundert ist demnach ein Jahrhundert des Mannes und des Vaters, der über intermediale Verweise, über Anspielungen auf das Christentum sowie über Hinweise auf den Puppenspieler die gesamte Szenerie beherrscht und die mechanischen Automaten zum Kindersatz macht. Der Grund dafür liegt auch darin, dass die Kinder nur noch bedingt den Vorgaben ihrer Eltern entsprechen wollen. So ist Kate Walker latent genervt, wenn sie mit ihrer Mutter telefoniert, und Hans Voralberg ist nur deswegen aus Valadilène geflohen, weil sein Vater mehr und mehr zum Tyrannen wurde. Der paternale Wunsch nach einer automatischen Produktion kindlicher Abbilder ihrer selbst geht allerdings auf narrativer Ebene nicht in Erfüllung, immerhin sind die Fabriken stillgelegt und die Federantriebe sind erschlafft.

21. Ibid.

22. Kenneth Branagh, Mary Shelley's Frankenstein, Tristar, 1994. 
Das Spiel nutzt so ein doppeltes Spannungsverhältnis: Zum einen zeigt es das Wechselverhältnis von Simulation und Repräsentation. Das Materiallager ist als Bild der Simulation ein ökonomischer Warenbestand, der nicht mehr abgerufen wird, und es ist gleichwohl eine Digitaltechnologie, die die patriarchale Produktionskultur zumindest dem Verdacht nach weiterschreiben könnte. Durch Repräsentation wird das Material als Mittel vergangener männlicher Schaffenskraft - insbesondere durch zahlreiche intermediale Verweise überhaupt erst sichtbar. Zum anderen entsteht hier ein Spannungsverhältnis zwischen der äußeren und der inneren Ansicht des Materials, so wie Böhme es skizziert hat. Äußerlich ist das Material durch Holz, Metall, Rost und Schnee ein zeitliches Maß der männlichen Schaffenskraft, das im Inneren der Textur gleich keine materielle Entsprechung mehr hat. Im zweiten Teil von Syberia ist dies sehr eindrücklich visualisiert: Hier soll der Zugführer Oskar dabei helfen, den stark geschwächten Hans Voralberg an das Ziel seiner Reise zu bringen. Dies geschieht, indem Kate Walker die Oberfläche des Automaten öffnet, wodurch sein Brustkorb und die Beine aufspringen. Völlig überraschend gibt es hier keine Mechanik im Inneren, vielmehr ist er leer, sodass sein Konstrukteur ihn als eine stabilisierende Rüstung verwenden kann. Die Automaten gleichen somit Texturen, die äußerlich die Anmutung eines Menschen haben, deren leeres Inneres aber freigelassen wird, um dem Menschen zu dienen. Im Fokus des Spiels steht somit der Mann des 20. Jahrhunderts, der seine Kinder - Kate Walker wie auch die Automaten - als Erfüllungsgehilfen bzw. Werkzeuge ansieht, mit Hilfe derer die angestoßenen Prozesse wieder in Gang gesetzt werden können. An der Stelle der inneren Ansicht steht somit die Leere der Kinder, die von den Vätern mit ihren Wünschen und Zielen befüllt wird. Dass diese Leere eine ökonomische ist, ist deshalb ersichtlich, weil die Kinder als hohle Werkzeuge verstanden und reflektiert werden, die als Mittel zum Zweck herhalten müssen.

\section{Das Material des Videospiels}

Syberia erzählt eine kritische Geschichte der Moderne, deren Zentralprinzipien das Patriarchat, der erschlaffende und neu aufgezogene Fortschritt und die Ökonomie sind. Dies geschieht, indem das Spiel seine eigenen Strukturen mit diesem Bild der Moderne parallelisiert: So sind der Spielprozess, der voranschreiten muss, die Simulation als Verwaltungstechnologie und als Grundlage der Darstellung der im Spiel semantisierten Materialien perfekte Strukturen, die eine solche Geschichte der Moderne überhaupt erst erzählbar machen. Die Väter sind dann die Auftraggeber, die Kinder die Erfüllungsgehilfen - oder die Spieler - und der erschlaffende Federmechanismus das sich selbst perpetuierende Spielziel. Das Spiel setzt sich dabei mit der leeren inneren Ansicht der Textur auseinander, indem es diese zu befüllen versucht und lotet somit das Spannungsverhältnis zwischen der äußeren und inneren Ansicht der Textur neu aus: Die äußere Ansicht ist als Marker vergehender Zeit gesetzt (Rost), wohingegen die innere Ansicht den kapitalistischen Menschen des 20. Jahrhunderts fokussiert. 
Die Analyse hat demnach gezeigt, dass, versöhnlich gesprochen, im Inneren digitaler Objekte nicht die Leere, sondern der Mensch herrscht, der das Spiel durch Interaktion überhaupt erst verlebendigt und den Federmechanismus wieder in Gang setzt. Weniger versöhnlich gesprochen ist dieser Mensch aber auch ein ökonomisierbares Mittel, das einzig dazu da ist, die von der vorherrschenden Umwelt vorgegebenen Ideale zu vertreten, um so die hier so kritisch betrachtete Moderne wieder in Gang zu setzen. Insofern wäre Syberia auch als eine kleine Kulturgeschichte des Videospiels zu lesen, die weniger narrativ als kapitalistisch und mechanisch motiviert ist und dabei das Material als gleichwohl simulatives, spielprozessuales, soziokulturelles und narratives Mittel einsetzt sowie fruchtbar macht. Dabei entzieht sich das Spiel immer wieder der Frage nach den Materialien des 21. Jahrhunderts, genauso wie der Frage nach der Materialität von Videospielen als solche. Hinter der Textur herrscht letztlich eben doch nur eine Leere, die positivistisch befüllt werden müsste, pessimistisch gesprochen aber Videospiele trotz ihrer relativen Neuheit als schon wieder veraltet, weil im 20. Jahrhundert verhaftet, ansieht. Wie Spiele in Zukunft mit dieser Kulturgeschichte umgehen und ob sie sich wie hoffentlich die Protagonistin im Spiel auch aus diesen Bezügen befreien können, muss sich erst noch erweisen. 